\title{
Hypercomplex Integrable Systems
}

\author{
J.D.E. Grant, I.A.B. Strachan \\ Department of Mathematics, University of Hull, \\ Hull, HU6 7RX, England. \\ e-mail: j.d.grant@maths.hull.ac.uk, i.a.strachan@maths.hull.ac.uk
}

\begin{abstract}
In this paper we study hypercomplex manifolds in four dimensions. Rather than using an approach based on differential forms, we develop a dual approach using vector fields. The condition on these vector fields may then be interpreted as Lax equations, exhibiting the integrability properties of such manifolds. A number of different field equations for such hypercomplex manifolds are derived, one of which is in Cauchy-Kovaleskaya form which enables a formal general solution to be given. Various other properties of the field equations and their solutions are studied, such as their symmetry properties and the associated hierarchy of conservation laws.
\end{abstract}

\section{Contents}

\begin{tabular}{llr}
\hline 1 & Introduction & 2
\end{tabular}

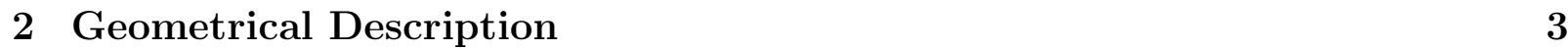

2.1 Hypercomplex geometry . . . . . . . . . . . . . . . . . . . 3

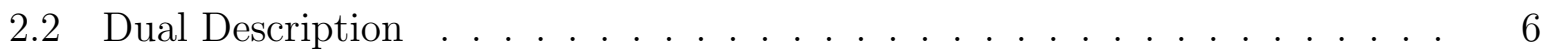

2.3 Local coordinate representations . . . . . . . . . . . . . . . . . . . . . . 8

2.3 .1 Case $1 . \ldots \ldots \ldots \ldots$

2.3.2 Case 11 . . . . . . . . . . . . . . . . . . . . . . . . . 10

2.3 .3 Case III . . . . . . . . . . . . . . . . . . . . . . . . . . . . . . 10

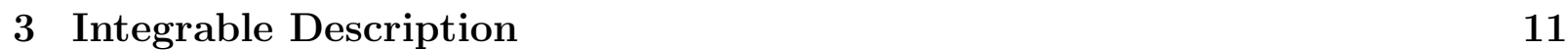

3.1 Lax Pair . . . . . . . . . . . . . . . . . . . . . . 11

3.2 Formal Solutions . . . . . . . . . . . . . . . . . . . . . . . . . 12

3.3 Symmetry Structure . . . . . . . . . . . . . . . . . . . . . . . . . . 12

3.4 Conservation Laws . . . . . . . . . . . . . . . . . . . . . . . . . 15

\begin{tabular}{llr}
\hline 4 & Comments & 16
\end{tabular} 


\section{Introduction}

The study of hyperKähler geometries has developed in two distinct directions. Starting with the work of Calabi [C] there has been a purely geometric vein, where such manifold are constructed and studied geometrically without reference to any defining set of field equations. The second vein starts with such field equations - systems of differential equations, and uses solutions of such systems to construct the manifolds.

To show how these two approaches are connected it is necessary to restrict one's attention to four dimensions, where the hyperKähler condition is equivalent to the existence of a metric with anti-self-dual Weyl tensor and vanishing Ricci tensor. Such metrics were shown by Penrose to have a corresponding twistor space, and conversely, that from such a twistor space one may reconstruct the metric. Such a structure appears at first sight to be special to four dimensions where there is the notion of self-duality, but it was shown in Sa that hyperKähler metric have corresponding twistor space in any $4 N$-dimensional space.

With more recent work, initiated by Ward [W] (see also [MW]), these two threads may be seen to be intimately interwoven. The existence of a (suitable) twistor space indicates that one is dealing with an integrable system. Thus any set of field equations for a hyperKähler metric provides one with an example of a multidimensional integrable system. From a solution to this integrable system one may construct the associated twistor space (and vica-versa), whose properties may be studied, and hence properties of the metric, without recourse to the particular differential equation, whose precise form depends on the particular coordinate representation being used.

The study of hypercomplex manifolds has had a similar history. In [FP] Finley and Plebański studied field equations which were derived from the following condition on the self-dual 2-form $\Sigma^{i}$,

$$
d \boldsymbol{\Sigma}^{i}=\boldsymbol{\alpha} \wedge \boldsymbol{\Sigma}^{i}, \quad i=1,2,3,
$$

though without mention of the associated complex geometry. Similarly Boyer [BO] studied the geometric aspects, but did not write down field equations for such metrics. In neither case was the link with integrable systems made. The aim of this paper is to show that one may write down systems of differential equations, solution of which define hypercomplex manifolds (here we will restrict out attention to four dimensions, though many of the ideas will generalize to $4 N$-dimensions). Since hypercomplex manifolds also have associated twistor spaces (in four dimensions the hypercomplex condition implies, though is not implied by, the anti-self-duality of the Weyl tensor [Bo] ) the field equations for these systems will be examples of multicomponent, multidimensional integrable systems.

Since hyperKähler metrics are obviously Kähler they may be written in terms of a single functions, the Kähler potential $\Omega$ :

$$
\mathbf{g}=\frac{\partial^{2} \Omega}{\partial x^{i} \partial \tilde{x}^{j}} d x^{i} d \tilde{x}^{y}, \quad i, j=12 .
$$


In four dimensions the hyperKähler conditions results in the differential equation

$$
\Omega_{x^{1} \tilde{x}^{1}} \Omega_{x^{2} \tilde{x}^{2}}-\Omega_{x^{1} \tilde{x}^{2}} \Omega_{x^{2} \tilde{x}^{1}}=1
$$

known as Plebański's equation. In this form it is hard (though not impossible) to apply ideas from the theory of integrable systems, which are best suited to evolutionary type equations. In [G1 the first author showed how, by performing a suitable Legendre transformation one may obtain an equation in evolutionary, or Cauchy-Kovaleskaya form, namely

$$
\psi_{t t}=\psi_{z t} \psi_{x y}-\psi_{y z} \psi_{x t}
$$

and the second author [S1] showed how to construct the associated integrable hierarchy, based on the study of the generalised symmetries of this equation.

In this paper the following two-component generalization of (1.1) will be studied:

$$
\begin{aligned}
& g_{t t}=\left\{g_{x}, g\right\}+\left\{h, g_{z}\right\}, \\
& h_{t t}=\left\{h_{x}, g\right\}+\left\{h, h_{z}\right\} .
\end{aligned}
$$

From the purely integrable systems aspect, considered in section 3, one may view this system as resulting from dropping the volume preserving condition on the vector fields which appear in the Lax pair used to construct (1.1). This approach will be followed in section 3. However, the system has a more geometric interpretation: solutions define hypercomplex metrics. This aspect will be considered in the next section. It should be pointed out that these hypercomplex structure are not the most general possible, but a particular subclass. The more general case will be considered in a future paper.

\section{Geometrical Description}

\subsection{Hypercomplex geometry}

In this subsection, we wish to investigate a special subclass of four-dimensional hypercomplex structures. In particular, assume we are working on a four-manifold $X$, and that we have a local basis for the tangent space, in the form of a set of four linearly-independent vector fields, $\left\{\mathbf{e}_{i}: i=1, \ldots, 4\right\}$. We wish to study the geometrical structures which arise if we assume that these vector fields obey the Lie-bracket relations:

$$
\begin{aligned}
& {\left[\mathbf{e}_{1}, \mathbf{e}_{2}\right]+\left[\mathbf{e}_{3}, \mathbf{e}_{4}\right]=0,} \\
& {\left[\mathbf{e}_{1}, \mathbf{e}_{3}\right]+\left[\mathbf{e}_{4}, \mathbf{e}_{2}\right]=0,} \\
& {\left[\mathbf{e}_{1}, \mathbf{e}_{4}\right]+\left[\mathbf{e}_{2}, \mathbf{e}_{3}\right]=0 .}
\end{aligned}
$$

We will show that in this case, the manifold $X$ is (locally) hyper-complex. Recall that a manifold $M$ of dimension $n=4 m$ is hyper-complex if it admits three integrable complex 
structures, I, J, K, which obey the quaternion multiplication relations:

$$
\begin{gathered}
\mathbf{I}^{2}=\mathbf{J}^{2}=\mathbf{K}^{2}=-\mathrm{Id}_{T_{x} M}, \\
\mathbf{I} \circ \mathbf{J}=\mathbf{K}, \quad \mathbf{J} \circ \mathbf{K}=\mathbf{I}, \quad \mathbf{K} \circ \mathbf{I}=\mathbf{J} .
\end{gathered}
$$

Such structures imply that the bundle of linear frames $L(M)$ reduces from a $G L(4 m, \mathbb{R})$ bundle to a $G L(m, \mathbb{H})$ bundle. In the special case of four dimensions, the frame bundle reduces to a $G L(1, \mathbb{H}) \cong \mathbb{H}^{*} \cong \mathbb{R}^{+} \times S U(2)$ bundle. Since $S U(2)$ is a subgroup of $S O(4)$, this means that in four dimensions, a hypercomplex structure automatically defines a conformal structure. In particular, there is a metric, unique up to a scale, with respect to which all the complex structures are Hermitian:

$$
\mathbf{g}(\mathbf{I X}, \mathbf{I Y})=\mathbf{g}(\mathbf{J X}, \mathbf{J Y})=\mathbf{g}(\mathbf{K X}, \mathbf{K Y})=\mathbf{g}(\mathbf{X}, \mathbf{Y}),
$$

where $\mathbf{X}, \mathbf{Y}$ are arbitrary sections of $T M$. The structures $\mathbf{I}, \mathbf{J}, \mathbf{K}$ along with a representative metric in the conformal structure, $\mathbf{g}$, define a hyper-hermitian structure. Each metric in this conformal structure has anti-self-dual Weyl tensor, with respect to the canonical orientation defined by any of the complex structures.

In the case we wish to study, we have a set of vector fields which satisfy the relations of equation (2.3). If we define the dual basis $\left\{\boldsymbol{\epsilon}^{i}\right\}$ for $T^{*} X$, then we will show that the vector fields define three integrable complex structures which obey the relations given in equation (2.4) and that the metrics with respect to which all these structures are hermitian are conformal to the metric:

$$
\mathrm{g}=\boldsymbol{\epsilon}^{1} \otimes \boldsymbol{\epsilon}^{1}+\boldsymbol{\epsilon}^{2} \otimes \boldsymbol{\epsilon}^{2}+\boldsymbol{\epsilon}^{3} \otimes \boldsymbol{\epsilon}^{3}+\boldsymbol{\epsilon}^{4} \otimes \boldsymbol{\epsilon}^{4} .
$$

The plan of the section is as follows. In the next section, we prove the assertions made above concerning the existence of hyper-complex structures, and a compatible conformal structure. Next the relation between this approach, based on vector fields, and the more usual approach based on forms is given. The vector field approach is useful for two reasons: firstly it enables field equations to be derived easily, and secondly it makes the connection with integrable systems more transparent. We then consider various coordinate versions of these equations.

Theorem 2.1 On a four-dimensional manifold $X$, if there exist four linearly-independent, non-vanishing vector fields $\left\{\mathbf{e}_{i}: i=1,2,3,4\right\}$ on a manifold $X$ which obey the Lie Bracket relations

$$
\begin{aligned}
& {\left[\mathbf{e}_{1}, \mathbf{e}_{2}\right]+\left[\mathbf{e}_{3}, \mathbf{e}_{4}\right]=0,} \\
& {\left[\mathbf{e}_{1}, \mathbf{e}_{3}\right]+\left[\mathbf{e}_{4}, \mathbf{e}_{2}\right]=0,} \\
& {\left[\mathbf{e}_{1}, \mathbf{e}_{4}\right]+\left[\mathbf{e}_{2}, \mathbf{e}_{3}\right]=0,}
\end{aligned}
$$

then the manifold is locally hypercomplex. Moreover, there exists a unique conformal structure, defined by the representative metric:

$$
\mathrm{g}=\boldsymbol{\epsilon}^{1} \otimes \boldsymbol{\epsilon}^{1}+\boldsymbol{\epsilon}^{2} \otimes \boldsymbol{\epsilon}^{2}+\boldsymbol{\epsilon}^{3} \otimes \boldsymbol{\epsilon}^{3}+\boldsymbol{\epsilon}^{4} \otimes \boldsymbol{\epsilon}^{4} .
$$

with respect to which all of the complex structures are Hermitian. 
Proof Consider maps $(\mathbf{I}, \mathbf{J}, \mathbf{K})$ on $T X$ defined by

$$
\begin{aligned}
& \mathbf{I}\left(\mathbf{e}_{1}\right)=+\mathbf{e}_{2}, \quad \mathbf{I}\left(\mathbf{e}_{2}\right)=-\mathbf{e}_{1}, \quad \mathbf{I}\left(\mathbf{e}_{3}\right)=+\mathbf{e}_{4}, \quad \mathbf{I}\left(\mathbf{e}_{4}\right)=-\mathbf{e}_{3}, \\
& \mathbf{J}\left(\mathbf{e}_{1}\right)=+\mathbf{e}_{3}, \quad \mathbf{J}\left(\mathbf{e}_{2}\right)=-\mathbf{e}_{4}, \quad \mathbf{J}\left(\mathbf{e}_{3}\right)=-\mathbf{e}_{1}, \quad \mathbf{J}\left(\mathbf{e}_{4}\right)=+\mathbf{e}_{2}, \\
& \mathbf{K}\left(\mathbf{e}_{1}\right)=+\mathbf{e}_{4}, \quad \mathbf{K}\left(\mathbf{e}_{2}\right)=+\mathbf{e}_{3}, \quad \mathbf{K}\left(\mathbf{e}_{3}\right)=-\mathbf{e}_{2}, \quad \mathbf{K}\left(\mathbf{e}_{4}\right)=-\mathbf{e}_{1} .
\end{aligned}
$$

To impose that these structures are integrable, we consider the complexification of the tangent space $T_{c} X=T X \otimes \mathbb{C}$. Consider first the structure $\mathbf{I}$. The almost complex structure leads to a direct sum decomposition of $T_{c} X$ as $T^{(1,0)} \oplus T^{(0,1)}$ where $\mathbf{v} \in T^{(1,0)}$ if $\mathbf{I}(\mathbf{v})=i \mathbf{v}$, and $\mathbf{v} \in T^{(0,1)}$ if $\mathbf{I}(\mathbf{v})=-i \mathbf{v}$. The complex structure is integrable iff the space $T^{(1,0)}$ is closed under the Lie bracket. In the case of the structure $\mathbf{I}, T^{(1,0)}$ is spanned by the vector fields $\left\{\mathbf{e}_{1}+i \mathbf{e}_{2}, \mathbf{e}_{3}+i \mathbf{e}_{4}\right\}$, and the only non-trivial Lie bracket we must consider is

$$
\left[\mathbf{e}_{1}+i \mathbf{e}_{2}, \mathbf{e}_{3}+i \mathbf{e}_{4}\right]=\left[\mathbf{e}_{1}, \mathbf{e}_{3}\right]+\left[\mathbf{e}_{4}, \mathbf{e}_{2}\right]+i\left(\left[\mathbf{e}_{1}, \mathbf{e}_{4}\right]+\left[\mathbf{e}_{2}, \mathbf{e}_{3}\right]\right) .
$$

For the right hand side to lie in $T^{(1,0)}$ implies a number of algebraic relations which, together with the analogous equations for $\mathbf{J}$ and $\mathbf{K}$ and the quaternionic relations, implies that

$$
\begin{aligned}
& {\left[\mathbf{e}_{1}, \mathbf{e}_{2}\right]+\left[\mathbf{e}_{3}, \mathbf{e}_{4}\right]=-A_{2} \mathbf{e}_{1}+A_{1} \mathbf{e}_{2}-A_{4} \mathbf{e}_{3}+A_{3} \mathbf{e}_{4},} \\
& {\left[\mathbf{e}_{1}, \mathbf{e}_{3}\right]+\left[\mathbf{e}_{4}, \mathbf{e}_{2}\right]=-A_{3} \mathbf{e}_{1}+A_{4} \mathbf{e}_{2}+A_{1} \mathbf{e}_{3}-A_{2} \mathbf{e}_{4},} \\
& {\left[\mathbf{e}_{1}, \mathbf{e}_{4}\right]+\left[\mathbf{e}_{2}, \mathbf{e}_{3}\right]=-A_{4} \mathbf{e}_{1}-A_{3} \mathbf{e}_{2}+A_{2} \mathbf{e}_{3}+A_{1} \mathbf{e}_{4}}
\end{aligned}
$$

for some set of functions $\left\{A_{1}, A_{2}, A_{3}, A_{4}\right\}$. Thus by construction the corresponding metric (2.5) is hypercomplex. It will be useful in what follows to combine these functions into a one form $\mathbf{A}=A_{i} \epsilon^{i}$ and regard this as a connection on $X$.

Unlike the conditions for a metric to be hyperKähler, the hypercomplex conditions are invariant under conformal changes of the metric. If $\mathbf{g} \rightarrow e^{2 \Lambda} \mathbf{g}$ then the corresponding transformation for the connection $\mathbf{A}$ is

$$
\mathbf{A} \rightarrow \mathbf{A}+2 d \Lambda .
$$

The subclass of hypercomplex structures which we are examining in this paper are defined by the conformal invariant condition $d \mathbf{A}=0$, i.e. the connection defined by $\mathbf{A}$ is flat. Thus locally one may define, for this subclass of hypercomplex structures, the conformal factor so that $\mathbf{A}=0$. This fixes the conformal structure.

It is a straightforward exercise using the explicit form of the complex structures given above to show that metric $\mathbf{g}$ given above is Hermitian with respect to each of the complex structures, and that it is the unique symmetric tensor (up to a rescaling) with this property.

The hypercomplex condition determines a metric up to a conformal factor. The zero curvature condition on the connection A may be used to fix the conformal factor. This determines the metric uniquely, up to trivial transformations. In terms of a null tetrad in which

$$
\mathrm{g}=\boldsymbol{\epsilon}^{1} \otimes_{S} \boldsymbol{\epsilon}^{2}+\boldsymbol{\epsilon}^{3} \otimes_{S} \boldsymbol{\epsilon}^{4}
$$


the conditions (2.4) become

$$
\begin{aligned}
{\left[\mathbf{e}_{1}, \mathbf{e}_{2}\right]+\left[\mathbf{e}_{3}, \mathbf{e}_{4}\right] } & =0 \\
{\left[\mathbf{e}_{1}, \mathbf{e}_{3}\right] } & =0 \\
{\left[\mathbf{e}_{2}, \mathbf{e}_{4}\right] } & =0
\end{aligned}
$$

This form will be used in later sections and is also used in the following example.

Example Consider the following vector fields

$$
\begin{array}{ll}
\mathbf{e}_{1}=\partial_{w} & \mathbf{e}_{2}=\left(1+|w|^{2}\right) \partial_{\bar{w}}+\bar{z} w \partial_{\bar{z}}, \\
\mathbf{e}_{4}=\partial_{z} & \mathbf{e}_{3}=\left(1+|z|^{2}\right) \partial_{\bar{z}}+z \bar{w} \partial_{\bar{w}} .
\end{array}
$$

It is easy to verify that these satisfy the conditions (2.6). Hence, by the above theorem, the corresponding Hermitian hypercomplex metric on $\mathbb{C}^{2}$ is conformal to

$$
\mathbf{g}=\left(1+|z|^{2}\right) d w d \bar{w}+\left(1+|z|^{2}\right) d z d \bar{z}-z \bar{w} d \bar{z}-\bar{z} w d z d \bar{w}
$$

Note, this metric is locally conformal to the Fubini-Study metric on $\mathbb{C P}^{2}$. However this construction does not extend from $\mathbb{C}^{2}$ to $\mathbb{C P}^{2}$. If it did it would contradict Boyer's [Bo] classification of compact hyperHermitian manifolds. Other examples, with tri-holomorphic Killing vectors, have been constructed in CTV, GT.

For the metric to be hyperKähler the corresponding Kähler forms defined by

$$
\begin{aligned}
\boldsymbol{\Omega}_{I}(X, Y) & =\mathbf{g}(I X, Y), \\
\boldsymbol{\Omega}_{J}(X, Y) & =\mathbf{g}(J X, Y), \\
\boldsymbol{\Omega}_{K}(X, Y) & =\mathbf{g}(K X, Y),
\end{aligned}
$$

must be closed, or equivalently, that $\nabla I=\nabla J=\nabla K=0$. It was shown in MN that these conditions are equivalent to the vector fields $\mathbf{e}_{i}$ being volume preserving, that is

$$
\mathcal{L}_{\mathrm{e}_{\mathrm{i}}} \boldsymbol{\omega}=0
$$

where $\boldsymbol{\omega}$ is some volume form.

\subsection{Dual Description}

The starting point for the study of four dimensional hypercomplex manifolds has traditionally been the equation

$$
d \boldsymbol{\Sigma}^{i}=\boldsymbol{\alpha} \wedge \boldsymbol{\Sigma}^{i}, \quad i=1,2,3
$$

where the $\Sigma^{i}$ are self-dual two forms on the manifold $X$. In this paper we have so far used a dual description, using vector fields rather than forms. This subsection is intended to 
bridge the gap between these two approaches. It is first necessary to fix some notation. The connection one-forms are defined by

$$
d \boldsymbol{\epsilon}^{i}+\Gamma_{j}^{i} \wedge \boldsymbol{\epsilon}^{j}=0
$$

so, in components, $\Gamma^{i}{ }_{j}=\Gamma^{i}{ }_{j k} \boldsymbol{\epsilon}^{k}$. The antisymmetric parts of $\Gamma^{i}{ }_{j k}$ are related to the structure functions defined by the Lie bracket $\left[\mathbf{e}_{j}, \mathbf{e}_{k}\right]=c_{j k}{ }^{i} \mathbf{e}_{i}$ by

$$
\Gamma_{[j k]}^{i}=\frac{1}{2} c_{j k}^{i}
$$

This formula enable one to connect these two approaches.

Proposition 2.1 The connection between equations 2.5 and (2.7) is given by the formulae

$$
\alpha=\mathbf{A}-\chi
$$

where

$$
\begin{aligned}
\mathbf{A} & =A_{i} \boldsymbol{\epsilon}^{i}, \\
\chi & =c_{i j}{ }^{j} \boldsymbol{\epsilon}^{i} .
\end{aligned}
$$

Proof Consider the self-dual two-form $\Sigma^{1}=\epsilon^{1} \wedge \epsilon^{2}+\epsilon^{3} \wedge \epsilon^{4}$. Then

$$
\begin{aligned}
d \boldsymbol{\Sigma}^{1} & =d\left(\boldsymbol{\epsilon}^{1} \wedge \boldsymbol{\epsilon}^{2}+\boldsymbol{\epsilon}^{3} \wedge \boldsymbol{\epsilon}^{4}\right) \\
& =-\Gamma_{[a b]}^{1} \boldsymbol{\epsilon}^{a} \wedge \boldsymbol{\epsilon}^{b} \wedge \boldsymbol{\epsilon}^{2}+\Gamma_{[a b]}^{2} \boldsymbol{\epsilon}^{a} \wedge \boldsymbol{\epsilon}^{b} \wedge \boldsymbol{\epsilon}^{1}-\Gamma_{[a b]}^{3} \boldsymbol{\epsilon}^{a} \wedge \boldsymbol{\epsilon}^{b} \wedge \boldsymbol{\epsilon}^{4}+\Gamma_{[a b]}^{4} \boldsymbol{\epsilon}^{a} \wedge \boldsymbol{\epsilon}^{b} \wedge \boldsymbol{\epsilon}^{3} \\
& =-\frac{1}{2} c_{a b}{ }^{1} \boldsymbol{\epsilon}^{a} \wedge \boldsymbol{\epsilon}^{b} \wedge \boldsymbol{\epsilon}^{2}+\frac{1}{2} c_{a b}{ }^{2} \boldsymbol{\epsilon}^{a} \wedge \boldsymbol{\epsilon}^{b} \wedge \boldsymbol{\epsilon}^{1}-\frac{1}{2} c_{a b}^{3} \boldsymbol{\epsilon}^{a} \wedge \boldsymbol{\epsilon}^{b} \wedge \boldsymbol{\epsilon}^{4}+\frac{1}{2} c_{a b}{ }^{4} \boldsymbol{\epsilon}^{a} \wedge \boldsymbol{\epsilon}^{b} \wedge \boldsymbol{\epsilon}^{3} \\
& =+\boldsymbol{\epsilon}^{1} \wedge \boldsymbol{\epsilon}^{2} \wedge\left(c_{a b}{ }^{a} \boldsymbol{\epsilon}^{b}+A_{3} \boldsymbol{\epsilon}^{3}+A_{4} \boldsymbol{\epsilon}^{4}\right)+\boldsymbol{\epsilon}^{3} \wedge \boldsymbol{\epsilon}^{4} \wedge\left(c_{a b}{ }^{a} \boldsymbol{\epsilon}^{b}+A_{1} \boldsymbol{\epsilon}^{1}+A_{2} \boldsymbol{\epsilon}^{2}\right) \\
& =\left(\boldsymbol{\epsilon}^{1} \wedge \boldsymbol{\epsilon}^{2}+\boldsymbol{\epsilon}^{3} \wedge \boldsymbol{\epsilon}^{4}\right) \wedge(\mathbf{A}-\boldsymbol{\chi}), \\
& =(\mathbf{A}-\boldsymbol{\chi}) \wedge \boldsymbol{\Sigma}^{1} .
\end{aligned}
$$

The manipulations for the remaining self-dual forms are identical

The hypercomplex condition on the metric is invariant under conformal changes of the metric. Thus one needs to calculate the transformation properties of these forms under such a change. 
Lemma 2.1 Under the conformal change $\mathbf{g} \rightarrow e^{2 \Lambda} \mathbf{g}$ the forms $\boldsymbol{\alpha}, \mathbf{A}, \boldsymbol{\chi}$ transform as

$$
\begin{aligned}
& \boldsymbol{\alpha} \rightarrow \boldsymbol{\alpha}+2 d \Lambda \\
& \mathbf{A} \rightarrow \mathbf{A}-d \Lambda \\
& \chi \rightarrow \chi-3 d \Lambda
\end{aligned}
$$

Proof The proof of this is entirely straightforward, following directly from the definition of the various forms. It is interesting to note that from these one may construct two linearly independent conformally invariant two forms.

Locally, if $d \boldsymbol{\alpha}=0$ then a conformal factor may be found so the resulting metric is hyperKähler. The metrics studied in this paper come from the conformally invariant condition $d \mathbf{A}=0$. This leaves another conformally invariant condition $d \boldsymbol{\chi}=0$, the significance of which will be investigated elsewhere.

\subsection{Local coordinate representations}

The aim of this section is to give some local coordinate descriptions of the equations (2.4). The main point that we make use of is that the equations (2.4) may be interpreted as the integrability conditions for null planes in the complexified tangent space of the manifold, and that we can introduce coordinates which naturally describe these surfaces.

With this approach in mind, we consider the complexified tangent bundle, $T_{c} X=$ $T X \otimes \mathbb{C}$. From the vector fields $\left\{\mathbf{e}_{i}\right\}$, we define the complex basis for $T_{c} X$ :

$$
\begin{aligned}
\mathbf{u} & =\mathbf{e}_{1}+i \mathbf{e}_{2}, \\
\mathbf{v} & =\mathbf{e}_{1}-i \mathbf{e}_{2}, \\
\mathbf{w} & =\mathbf{e}_{3}+i \mathbf{e}_{4}, \\
\mathbf{x} & =\mathbf{e}_{3}-i \mathbf{e}_{4} .
\end{aligned}
$$

In the case where we are considering the complexification of a real Riemannian structure, we would impose the reality condition that $\mathbf{u}$ and $\mathbf{v}$ would be related by complex conjugation, as would $\mathbf{w}$ and $\mathbf{x}$. More generally, we require the existence of a real structure on $T_{c} X$ to define real slices of different signatures. Since we will be interested in metrics of both Riemannian and ultra-hyperbolic signatures, we will generally assume, for the moment, that the vector fields $\mathbf{u}, \mathbf{v}, \mathbf{w}, \mathbf{x}$ are complex, and simply impose the relevant reality conditions later.

In terms of the vector fields $\mathbf{u}, \mathbf{v}, \mathbf{w}, \mathbf{x}$, the relations (2.4) take the form:

$$
\begin{gathered}
{[\mathbf{u}, \mathbf{w}]=0,} \\
{[\mathbf{u}, \mathbf{v}]+[\mathbf{w}, \mathbf{x}]=0,} \\
{[\mathbf{v}, \mathbf{x}]=0 .}
\end{gathered}
$$


The first of these equations states that at each point (on a suitable local neighbouhood of) $X$, the plane spanned by the vector fields $\mathbf{u}$ and $\mathbf{w}$ is integrable. It follows that we can introduce coordinates $(t, y)$ on each of these surfaces with

$$
\mathbf{u}=\partial_{t}, \quad \mathbf{w}=\partial_{y}
$$

If we now consider the space with $t$ and $y$ held constant, the last of equations (3.10) tells us that this space forms an integrable plane as well. As such, we can introduce coordinates $(x, z)$ on these planes, and the coordinates $(t, x, y, z)$ should give a suitable coordinate system on some local region of $X$. Note that the coordinates $(x, z)$ are not fully determined by these conditions. The different choices of coordinates correspond to different coordinate expressions for the vector fields $\mathbf{v}$ and $\mathbf{x}$, and in fact there are several geometrically distinct coordinate systems that we wish to investigate:

\subsubsection{Case I}

The first case we consider is where we take the coordinates $(x, z)$ to be null. This is the analogue of the usual complex coordinate description of Kahler metrics. Using the second of equations (3.10), we introduce functions $a$ and $b$ and let $\mathbf{v}$ and $\mathbf{x}$ take the form

$$
\mathbf{v}=a_{y} \partial_{x}-b_{y} \partial_{z}, \quad \mathbf{x}=-a_{t} \partial_{x}+b_{t} \partial_{z} .
$$

This is the generalisation of the form used in [CMN for the case of half-flat metrics. The functions $a$ and $b$ must now satisfy the equation

$$
\left\{a, a_{x}\right\}=\left\{b, a_{z}\right\}, \quad\left\{a, b_{x}\right\}=\left\{b, b_{z}\right\},
$$

where we have defined the Poisson Bracket by

$$
\{f, g\}=f_{t} g_{y}-f_{y} g_{t} .
$$

Equations (2.8) are a generalisation of the First Heavenly equation which describes halfflat metrics $[\mathrm{P}]$. To see this, we note that the vectors in (2.4) define a metric which is conformal to a half flat metric if the vectors $\mathbf{e}_{i}$ are divergence free with respect to some volume element $\boldsymbol{\omega} \mathbb{\mathbb { M N }}$. Taking $\boldsymbol{\omega}=d t \wedge d x \wedge d y \wedge d z$, we find that there exists a function $\Omega$ such that $a=\Omega_{z}, b=\Omega_{y}$. We can therefore integrate equations (2.8) once and rescale our coordinates $(y, z)$ such that $\Omega$ satisfies the equation

$$
\left\{\Omega_{y}, \Omega_{z}\right\}=1
$$

which is the First Heavenly equation $\mathbb{P} 1]$.

In this case, we may reconstruct the metric, $\mathrm{g}$, which takes the form:

$$
\mathbf{g}=4\left(b_{t} a_{y}-a_{t} b_{y}\right)^{-1}\left[d t \otimes\left(a_{t} d z+b_{t} d x\right)+d y \otimes\left(a_{y} d z+b_{y} d x\right)\right] .
$$

We see that in this form, the coordinates $(t, x, y, z)$ are all null, and are tailored to the geometry of the spheres worth of integrable null planes through each point of the space.

The metric is Riemannian if we assume that the functions $a$ and $b$ are real, and that the coordinates obey some reality condition. If the functions and the coordinates are real, the metric will be of ultra-hyperbolic signature. 


\subsubsection{Case II}

Here we introduce functions $\phi$ and $\psi$ and take

$$
\mathbf{v}=\partial_{x}+\phi_{y} \partial_{t}-\psi_{y} \partial_{y}, \quad \mathbf{x}=\partial_{z}-\phi_{t} \partial_{t}+\psi_{t} \partial_{y}
$$

The functions $\phi$ and $\psi$ must now satisfy the equations

$$
\begin{aligned}
& \phi_{t x}+\phi_{y z}+\left\{\phi_{t}, \phi\right\}+\left\{\psi, \phi_{y}\right\}=0 \\
& \psi_{t x}+\psi_{y z}+\left\{\psi_{t}, \phi\right\}+\left\{\psi, \psi_{y}\right\}=0
\end{aligned}
$$

In terms of these coordinates and functions, the metric is

$$
\mathbf{g}=4\left[\left(d t+\phi_{t} d z-\phi_{y} d x\right) \otimes d x+\left(d y-\psi_{t} d z+\psi_{y} d x\right) \otimes d z\right] .
$$

In this case, the coordinates $(t, y)$ are again null labelling some of the null planes in the space. The coordinates $(x, z)$ are not null, however, and label the "rate of change" of the null planes (see [NPT] for more on the geometrical interpretation of these coordinates.)

These equations have been previously investigated in connection with anti-self-dual structures [FP], and are a direct generalisation of the Second Heavenly Equation for halfflat metrics $[\mathbb{P}]$.

\subsubsection{Case III}

Finally, we consider the analogue of the expansion used in to reduce the half-flat case to evolution form [G1]. We therefore introduce functions $g$ and $h$ such that

$$
\mathbf{v}=\partial_{t}+g_{y} \partial_{x}-h_{y} \partial_{z}, \quad \mathbf{x}=-g_{t} \partial_{x}+h_{t} \partial_{z}
$$

We find that the functions $f$ and $g$ must satisfy the equations

$$
g_{t t}+\left\{g_{x}, g\right\}+\left\{h, g_{z}\right\}=0, \quad h_{t t}+\left\{h_{x}, g\right\}+\left\{h, h_{z}\right\}=0 .
$$

In this coordinate system, the metric takes the local form

$$
\mathrm{g}=4 \Delta^{-1}\left[d t \otimes\left(g_{t} d z+h_{t} d x\right)+d y \otimes\left(g_{y} d z+h_{y} d x\right)-\Delta^{-1}\left(g_{t} d z+h_{t} d x\right)^{2}\right]
$$

with $\Delta=\left(h_{t} g_{y}-g_{t} h_{y}\right)$. In this system, the coordinates $(t, y)$ still span null planes, however the geometrical interpretation of the $(x, z)$ coordinates is not particularly clear. The main advantage of this coordinate system, however, is that the equations of motion are in evolution form. This form of the equations is therefore the natural starting point for the study of symmetry algebras [G1], and the associated integrable hierarchy [S1]. We shall therefore concentrate on this form of the equations from now on. 


\section{Integrable Description}

In this section the system (1.2) will be studied, viewing it as an integrable system and hence applying various known results from the theory of integrable systems to it. In particular a Lax pair will be given for the system, a hierarchy of conservation laws constructed and the Lie-point symmetry structure calculated.

\subsection{Lax Pair}

A characteristic feature of an integrable system is the ability to express it as the compatibility condition for an otherwise overdetermined linear system. To obtain the system (1.2) in such a way consider the following vector fields on complexified tangent bundle, $T_{c} X=T X \otimes \mathbb{C}$ of a four manifold $X$ :

$$
\begin{aligned}
\mathbf{u} & =\partial_{t}, & \mathbf{w} & =\partial_{y}, \\
\mathbf{v} & =\partial_{t}+g_{y} \partial_{x}-h_{y} \partial_{z}, & \mathbf{x} & =-g_{t} \partial_{x}+h_{t} \partial_{z} .
\end{aligned}
$$

With these define the new vector fields

$$
\begin{aligned}
& \mathcal{L}_{0}=\mathbf{u}-\lambda \mathbf{x}, \\
& \mathcal{L}_{1}=\mathbf{w}+\lambda \mathbf{v},
\end{aligned}
$$

where $\lambda \in \mathbb{C P}^{1}$ is an auxiliary parameter. The compatibility conditions for the otherwise overdetermined linear system $\mathcal{L}_{0} \Psi=\mathcal{L}_{1} \Psi=0$ results in the following system of equations:

$$
\begin{gathered}
{[\mathbf{u}, \mathbf{w}]=0,} \\
{[\mathbf{u}, \mathbf{v}]+[\mathbf{w}, \mathbf{x}]=0,} \\
{[\mathbf{v}, \mathbf{x}]=0 .}
\end{gathered}
$$

With the explicit vector fields given above these reduce to the system (1.2):

$$
\begin{aligned}
g_{t t} & =\left\{g_{x}, g\right\}+\left\{h, g_{z}\right\}, \\
h_{t t} & =\left\{h_{x}, g\right\}+\left\{h, h_{z}\right\},
\end{aligned}
$$

where, for convenience, the Poisson bracket $\{f, g\}=f_{y} g_{z}-f_{z} g_{y}$ has been used. In the previous section the geometry underlying this construction was given; solutions define a hypercomplex metric on the manifold $X$. Here we just consider the system as an example of a four dimensional integrable system and study it thus.

One interesting reduction of this system is to impose the condition $g_{y}=h_{z}$. One may solve this constraint by introducing a function $\psi$ such that $h=\psi_{y}, g=\psi_{z}$. With this it is possible to integrate (1.2) and obtain a single evolution equation (1.1). With this constraint the basic vector fields $\mathbf{e}_{\mathbf{i}}=(\mathbf{u}, \mathbf{v}, \mathbf{w}, \mathbf{x})$ become volume preserving, that is

$$
\mathcal{L}_{\mathrm{e}_{\mathrm{i}}} \boldsymbol{\omega}=0
$$


where $\boldsymbol{\omega}$ is the volume form $\boldsymbol{\omega}=\mathbf{u} \wedge \mathbf{v} \wedge \mathbf{w} \wedge \mathbf{x}$. In this case the metric is hyperKähler rather than hypercomplex, with $\psi$ being related to the Kähler potential by a Legendre transformation. Conversely, one may regard the system (1.2) as a generalization of (1.1) when one relaxes the volume preserving condition on the vector fields in the associated Lax pair.

\subsection{Formal Solutions}

The system (1.1) is in Cauchy-Kovaleskaya form, so their formal solution may be written as a power series in the $t$-variable:

$$
\begin{aligned}
h & =\sum_{n=0}^{\infty} h_{n}(x, y, z) t^{n}, \\
g & =\sum_{n=0}^{\infty} g_{n}(x, y, z) t^{n}
\end{aligned}
$$

and the differential equations reduce to recursion relations between the coefficients $h_{n}, g_{n}$. Thus a formal solution may be derived from the coefficients $\left(g_{0}, h_{0}, g_{1}, h_{1}\right)$, or equivalently, in terms of the initial data $\left.\left(g, h, g_{t}, h_{t}\right)\right|_{t=0}$ on the $t=0$ hypersurface in $X$. This shows that the general solution depends on four arbitrary functions of three variables.

One simple, but explicit, solution may be obtained from the ansatz

$$
\begin{aligned}
& g=t y+G(t, x, z), \\
& h=t .
\end{aligned}
$$

With this the nonlinearites in (1.1) disappear and one is left with a linear equation for $G$, which after a simple change of variable, is just the three dimensional Laplace equation. Other simple solutions may be obtained by taking known hypercomplex metrics and reex-

pressing them in the above form. Some examples of solutions obtained in this way will be given later.

\subsection{Symmetry Structure}

While symmetry techniques may be applied to any system of differential equations, the Lie-point symmetries of integrable systems have a particularly rich structure compared to non-integrable systems. Indeed, possible integrable systems may often be indentified by an increase in the dimension of the Lie-algebra of symmetries, as compared to nearby non-integrable systems. 
Let $x=\left(x_{1}, \ldots, x_{p}\right)$ and $u=\left(u^{1}, \ldots, u^{q}\right)$ be sets of independent and dependent variables, and consider a set of differential equations of degree $k$, given by

$$
\Delta^{i}\left(x, u^{(k)}\right)=0, \quad i=1, \ldots, m .
$$

Lie-point symmetries are generated by the vector field

$$
\mathbf{v}=\sum_{i=1}^{p} \xi_{i}(x, u) \frac{\partial}{\partial x_{i}}+\sum_{\alpha=1}^{q} \phi_{\alpha}(x, u) \frac{\partial}{\partial u^{\alpha}},
$$

where the coefficients are determined by the criterion

$$
\left.\operatorname{pr}^{(k)} \mathbf{v}(\Delta)\right|_{\Delta=0}=0
$$

where $p r^{(k)} \mathbf{v}$ is the $k$-th prolongation of the vector field $\mathbf{v}$. These ideas, and notation, are standard, see [O].

To apply such a procedure to the system (1.2) it is convenient to convert the system from a second order system in two independent variables to a first order system in four independent variables by introducing potentials and integrability conditions. Therefore let:

$$
A=g_{t}, B=g_{y}, C=h_{t}, D=h_{y},
$$

in which $(1.2)$ becomes

$$
\begin{aligned}
A_{y}-B_{t} & =0 \\
C_{y}-D_{t} & =0 \\
A_{t}+A_{x} B-B_{x} A+C B_{z}-D A_{z} & =0 \\
C_{t}+C_{x} B-D_{x} A+C D_{z}-C_{z} D & =0
\end{aligned}
$$

Since these are first order the calculation of the first prolongation of the vector field is easy, the evolutionary form of the system also giving a distinguished variable $t$ to eliminate in the course of the calculations.

The result is that we get five families of symmetries, the vector fields which generate these families being (where $k$ is a constant, $\phi, \psi$ are functions of the coordinate $y$, and $a_{1}, a_{2}$ are functions of coordinates $\left.(x, z)\right)$ :

$$
\begin{aligned}
\mathbf{v}^{1}[k] & =k\left(-t \partial_{t}+2 A \partial_{A}+B \partial_{B}+2 C \partial_{C}+D \partial_{D}\right), \\
\mathbf{v}^{2}[\phi] & =-\phi \partial_{t}+A \phi_{y} \partial_{B}+C \phi_{y} \partial_{D}, \\
\mathbf{v}^{3}[\psi] & =-t \psi_{y} \partial_{t}-\psi \partial_{y}+\psi_{y}\left(A \partial_{A}+B \partial_{B}+C \partial_{C}+D \partial_{D}\right)+t \psi_{y y}\left(A \partial_{B}+C \partial_{D}\right), \\
\mathbf{v}^{4}\left[a_{1}\right] & =-a_{1} \partial_{x}-\left(a_{1 x} A-a_{1 z} C\right) \partial_{A}-\left(a_{1 x} B-a_{1 z} D\right) \partial_{B}, \\
\mathbf{v}^{5}\left[a_{2}\right] & =-a_{2} \partial_{z}-\left(a_{2 z} C-a_{2 x} A\right) \partial_{C}-\left(a_{2 z} D-a_{2 x} B\right) \partial_{D} .
\end{aligned}
$$

The first of these generators simply generates a scaling symmetry of the equations. Therefore, if $A, B, C, D$ constitute a solution of the equations, then so do $\tilde{A}, \tilde{B}, \tilde{C}, \tilde{D}$ defined 
by

$$
\begin{aligned}
& \tilde{A}(t, x, y, z)=\mathrm{e}^{2 k} A\left(\mathrm{e}^{k} t, x, y, z\right), \\
& \tilde{B}(t, x, y, z)=\mathrm{e}^{k} B\left(\mathrm{e}^{k} t, x, y, z\right), \\
& \tilde{C}(t, x, y, z)=\mathrm{e}^{2 k} C\left(\mathrm{e}^{k} t, x, y, z\right), \\
& \tilde{D}(t, x, y, z)=\mathrm{e}^{k} D\left(\mathrm{e}^{k} t, x, y, z\right) .
\end{aligned}
$$

Similarly, the second generator generates a translation in the $t$ coordinate along with a redefinition of fields, so that in this case

$$
\begin{aligned}
& \tilde{A}(t, x, y, z)=A(t+\phi, x, y, z), \\
& \tilde{B}(t, x, y, z)=B(t+\phi, x, y, z)+\phi_{y} A(t+\phi, x, y, z), \\
& \tilde{C}(t, x, y, z)=C(t+\phi, x, y, z), \\
& \tilde{D}(t, x, y, z)=D(t+\phi, x, y, z)+\phi_{y} C(t+\phi, x, y, z) .
\end{aligned}
$$

These are the only symmetries that it is possible to generally exponentiate explicitly. The non-zero commutators for this Lie algebra are:

$$
\begin{aligned}
{\left[\mathbf{v}^{1}[k], \mathbf{v}^{2}[\phi]\right] } & =\mathbf{v}^{2}[k \phi] \\
{\left[\mathbf{v}^{2}[\phi], \mathbf{v}^{3}[\psi]\right] } & =\mathbf{v}^{2}\left[\psi \phi_{y}-\phi \psi_{y}\right] \\
{\left[\mathbf{v}^{3}[\psi], \mathbf{v}^{3}[\chi]\right] } & =\mathbf{v}^{3}\left[\chi \psi_{y}-\psi \chi_{y}\right] \\
{\left[\mathbf{v}^{4}[a], \mathbf{v}^{4}[b]\right] } & =\mathbf{v}^{4}\left[a_{x} b-a b_{x}\right] \\
{\left[\mathbf{v}^{4}[a], \mathbf{v}^{5}[b]\right] } & =\mathbf{v}^{4}\left[a_{z} b\right]-\mathbf{v}^{5}\left[a b_{x}\right] \\
{\left[\mathbf{v}^{5}[a], \mathbf{v}^{5}[b]\right] } & =\mathbf{v}^{5}\left[a_{z} b-a b_{z}\right]
\end{aligned}
$$

where $k, l$ are arbitrary constants, $\psi, \phi$ are arbitrary functions of $y$, and $a, b$ are arbitrary functions of $x$ and $z$.

From the structure of these commutators one may decompose the Lie algebra $L$ generated by these vector fields into a direct sum $L=L_{1} \oplus L_{2}$, where

$$
\begin{aligned}
& L_{1}=\left\{\mathbf{v}^{1}[k], \mathbf{v}^{2}[\phi], \mathbf{v}^{3}[\psi]\right\}, \\
& L_{2}=\left\{\mathbf{v}^{4}[a], \mathbf{v}^{5}[b]\right\} .
\end{aligned}
$$

The sub-Lie-algebra $L_{1}$ decomposes further as a semidirect product $L_{1}=S \triangleright R$ where

$$
\begin{aligned}
S & =\left\{\mathbf{v}^{3}[\psi]\right\}, \\
R & =\left\{\mathbf{v}^{1}[k], \mathbf{v}^{2}[\phi]\right\} .
\end{aligned}
$$

The subalgebra $L_{2}$ is isomorphic to vector fields on a 2-dimensional surface and correspond to coordinate transformation in the $x, z$-variables. This is to be expected, since in the hyper-Kähler case the vectors are all divergence free and the symmetries turn out to be related to symplectic diffeomorphisms of two dimensional planes. In our case, we have simply dropped the divergence-free condition from the vector fields, and the symmetry group becomes related to the larger group of diffeomorphisms, since there is no natural symplectic structure any more. Similarly the vector fields $\mathbf{v}^{2}$ generate coordinate transformations and the vector fields $\mathbf{v}^{1}$ generate constant rescalings of the metric. The only vector fields which generate genuinely new metrics are those in $S$. 


\subsection{Conservation Laws}

In this section a hierarchy of conservation laws of the form

$$
g^{\mu \nu} \nabla_{\mu} j_{\nu}^{(n)}=0, \quad n=0,1, \ldots
$$

will be constructed. This expression is clearly covariant, but in the calculations it will be necessary to use a particular form of the metric and the associated field equations. Explicitly we consider metrics of the form

$$
\mathbf{g}=2 \Delta^{-\frac{1}{2}}\left[d t \otimes\left(a_{t} d z+b_{t} d x\right)+d y \otimes\left(a_{y} d z+b_{y} d x\right)\right]
$$

where $\Delta=\left(a_{t} b_{y}-a_{y} b_{t}\right)$ and with $a$ and $b$ being solutions of the field equations (2.8). The conformal fact in (3.12) has been fixed so that det $g_{i j}=1$; such a fixing does not change the hypercomplex or Hermitian properties of the metric. One obvious extension of these results would be to introduce the notion of a conformally invariant conservation law.

The starting point of this construction, a generalization of a procedure first applied to nonlinear $\sigma$-models [BIZZ, [L], is the solution $\Psi$ to the Lax pair

$$
\begin{aligned}
{\left[\lambda \partial_{t}+a_{t} \partial_{x}-b_{t} \partial_{z}\right] \Psi } & =0, \quad \lambda \in \mathbb{C P}^{1} \\
{\left[\lambda \partial_{y}+a_{y} \partial_{x}-b_{x} \partial_{z}\right] \Psi } & =0, \quad \text {. }
\end{aligned}
$$

Expanding $\Psi$ as a power series in $\lambda$, i.e. as $\Psi=\sum_{n=0}^{\infty} \lambda^{n} \Psi_{n}$ and equating coefficients yields the following equations for the $\Psi_{0}$-term:

$$
\begin{aligned}
{\left[a_{t} \partial_{x}-b_{t} \partial_{z}\right] \Psi_{0} } & =0 \\
{\left[a_{y} \partial_{x}-b_{y} \partial_{z}\right] \Psi_{0} } & =0
\end{aligned}
$$

and the recursion relations

$$
\begin{aligned}
\partial_{t} \Psi_{n} & =\left(-a_{t} \partial_{x}+b_{t} \partial_{z}\right) \Psi_{n+1}, \\
\partial_{y} \Psi_{n} & =\left(-a_{y} \partial_{x}+b_{y} \partial_{z}\right) \Psi_{n+1}
\end{aligned}
$$

The first set of equations imply that $\Psi_{0}=\Psi_{0}(t, y)$ and so we take the seed solution to be

$$
\Psi_{0}=\left(\begin{array}{c}
t \\
y
\end{array}\right)
$$

(here we have assembled two independent solutions into a vector). This seed solution will generate, via the recursion relations (3.15) the full solution to the Lax pair (3.13). This function defines the twistor surfaces in the corresponding twistor space. Another family of conservation laws may be obtained starting from the expansion $\tilde{\Psi}=\sum_{n=0}^{\infty} \lambda^{-n} \tilde{\Psi}_{n}$ and the relationship between $\Psi$ and $\tilde{\Psi}$ on the equator of $\mathbb{C P}^{1}$ defines the twistor space, via a patching construction NPT, S2]. 
Proposition 3.1 The currents $j_{\mu}^{(n)}$ defined by

$$
\begin{array}{ll}
j_{t}^{(n)}=0, & j_{x}^{(n)}=\Delta^{\frac{1}{2}} \partial_{x} \Psi_{n+1}, \\
j_{y}^{(n)}=0, & j_{z}^{(n)}=\Delta^{\frac{1}{2}} \partial_{z} \Psi_{n+1},
\end{array}
$$

are conserved.

Proof With the particular metric (3.12)

$$
\begin{aligned}
g^{\mu \nu} \nabla_{\mu} j_{\nu}^{(n)} & =\partial_{t}\left[-a_{y} \partial_{z} \Psi_{n+1}+b_{y} \partial_{z} \Psi_{n+1}\right]+\partial_{y}\left[+a_{t} \partial_{z} \Psi_{n+1}-b_{t} \partial_{z} \Psi_{n+1}\right] \\
& =\partial_{t}\left[\partial \Psi_{n}\right]-\partial_{y}\left[\partial_{t} \Psi_{n}\right] \\
& =0
\end{aligned}
$$

This proof uses the condition $\operatorname{det} g_{i j}=1$, so the Christoffel symbols $\Gamma_{\nu \mu}^{\mu}=0$.

\section{Comments}

Underlying the integrability of the multidimensional systems presented here is the existence of a twistor space. This paper has, though, only concentrated on the field equations and the associated Lax pairs with little mention of the properties of the corresponding twistor space - in terms of a double fibration

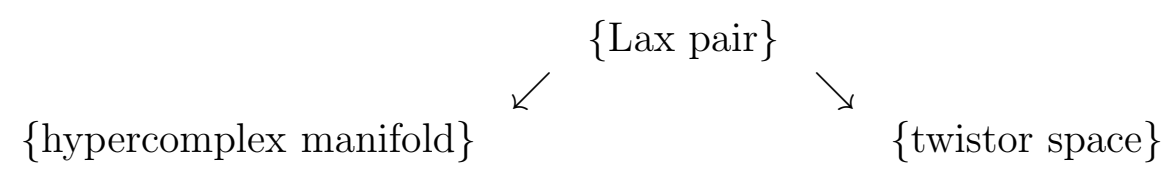

we have said little about about the structure of the right hand side. Such twistor space have the special property that they fibre over $\mathbb{C P}^{1}[\mathbb{B d}]$, unlike those for more general anti-selfdual Weyl spaces or scalar flat Kähler spaces. This is manifested in the simple $\lambda$-dependence in the Lax pairs for hypercomplex manifolds - the Lax pairs for scalar flat Kähler $\mathbb{P a}$ and general anti-self-dual Weyl spaces [G2, MW] involve $\partial_{\lambda}$-terms. The hypercomplex manifolds studied here come from the conformally invariant condition $d \mathbf{A}=0$, and so one would expect the corresponding twistor space to exhibit certain extra properties. General hypercomplex manifolds (without this condition) will be studied in the sequel to this paper, this also containing the connection between the approach developed here and the Obata connection.

One characteristic feature of integrable systems is the existence of an associated hierarchy. Such hierarchies may be constructed by studying the generalized symmetry structure of the original systems of equations [0]. Such hierarchies have been constructed for hyperKähler metrics in [52]. It remains to see how such ideas may be extended to the hypercomplex systems studied here. 


\section{Acknowledgments}

Financial support was provided by the EPSRC.

\section{Appendix}

While many of the ideas in this paper will generalize to $4 N$-dimensional manifolds, when $N=1$ another generalization is possible. Underlying the integrability of the structures studied in this paper is the existence of a suitable twistor space, and hypercomplex manifolds automatically have such twistor spaces. However in four dimensions the existence of a twistor space follows from the Weyl tensor being anti-self-dual, and the hypercomplex condition here implies, not is implied by, this condition. Thus a possible generalization is to study metrics with anti-self-dual Weyl tensor and which are also scalar flat. Such a system has an other reduction to scalar flat Kähler metrics. These different systems and their interconnections are summarized in the following diagram:

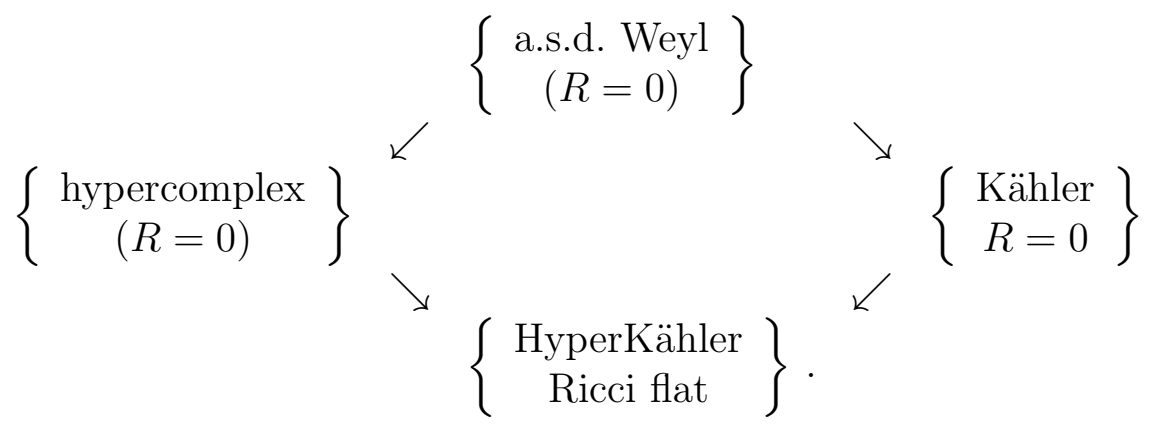

The conditions $(R=0)$ in brackets indicate how the conformal factor for otherwise conformally invariant conditions have been fixed.

An analogous system of equations to (1.1) for the scalar-flat, anti-self-dual-Weyl systems is given by

$$
\begin{aligned}
g_{t t}+\left(e^{-\psi}\{g, h\}\right)_{z} & =e^{-\psi}\left\{g, h_{z}-g_{y}\right\} \\
h_{t t}+\left(e^{-\psi}\{g, h\}\right)_{y} & =e^{-\psi}\left\{h, h_{z}-g_{y}\right\} \\
\left(e^{+\psi}\right)_{t t}+\left\{g, \psi_{y}\right\}-\left\{h, \psi_{z}\right\} & =0
\end{aligned}
$$

(these certainly imply the geometric conditions though whether they are implied by them is unclear). The corresponding metric is given by

$$
\mathbf{g}=+2 d y\left\{h_{t} d t+h_{x} d x-\frac{h_{t} e^{\psi}}{\Delta}\left[h_{t} d y+g_{t} d z\right]\right\}+2 d z\left\{g_{t} d t+g_{x} d x-\frac{g_{t} e^{\psi}}{\Delta}\left[h_{t} d y+g_{t} d z\right]\right\} .
$$

where $\Delta=h_{t} g_{x}-g_{t} h_{x}$. The two reductions above are easy to see from this system: 
- when $\psi=0$, this system reduces to

$$
\begin{aligned}
& g_{t t}=\left\{h, g_{z}\right\}-\left\{g, g_{y}\right\}, \\
& h_{t t}=\left\{h, h_{z}\right\}-\left\{g, h_{y}\right\},
\end{aligned}
$$

that is, to the hypercomplex systems studied in the main body of this paper. The further reduction $h=\theta_{y}, g=\theta_{z}$ reduces this down to the hyperKähler equation (1.1) .

- imposing the Kähler condition on this system gives $h=\theta_{y}, g=\theta_{z}$ and the first set of equations simplify to

$$
\begin{aligned}
\theta_{t t}+e^{-\psi}\left\{\theta_{z}, \theta_{y}\right\} & =0, \\
\left(e^{+\psi}\right)_{t t}+\left\{\theta_{z}, \psi_{y}\right\}-\left\{\theta_{y}, \psi_{z}\right\} & =0 .
\end{aligned}
$$

These are the analogues of the well-known scalar flat Kähler equations [E], written in evolutionary form. It is easy to find solutions, such as the one which gives the Burns metric. The further reduction $\psi=0$ reduces this down to the hyperKähler equation (1.1) .

One interesting class of solutions to all these systems comes from imposing an $S U(2)$ symmetry on the metrics. Such metrics are often referred to as Bianch IX metrics. This symmetry reduces the field equations from partial differential equations down to systems of coupled ordinary differential equations which may be integrated directly. These ideas may also be applied to other Bianchi metrics.

\section{References}

[BIZZ] Brézin, E., Itzykson, C., Zinn-Justin, J. and Zuber, J.B., Remarks about the existence of non-local charges in two dimensional models, Phys. Lett. 82B 442-451 (1979)

[Bo] Boyer, C.P., A note on hyperhermitian four-manifolds. Proceedings of the American Mathematical Society 102, 157-164 (1988).

[C] Calabi, E., Métriques kahlériennes et fibrés holomorphes, Ann. Ec. Norm. Sup. Paris 12 269-294 (1979).

[CMN] Chakravarty, S., Mason, L.J. and Newman E.T., Canonical structures on anti-selfdual four-manifolds and the diffeomorphism group, Journal of Mathematical Physics 32, 1458-1464 (1991). 
[CTV] Chave, T., Tod, K.P. and Valent, G., $(4,0)$ and $(4,4)$ sigma models with a triholomorphic Killing vector, Phys. Lett. B383 262-270 (1996).

[F] Flaherty, E.J., The Nonlinear Graviton in Interaction with a Photon, Gen. Rel. Grav. 9 961-978 (1978).

[FP] Finley, J.D. and Plebański, J.F., Further heavenly metrics and their symmetries, Journal of Mathematical Physics 17, 585-596 (1976).

[G1] Grant, J.D.E., On self-dual gravity, Physical Review D47, 2606-2612 (1993).

[G2] Grant, J.D.E., Some properties of anti-self-dual conformal structures, preprint 1998.

[GT] Gauduchon, P. and Tod, K.P., Hyper-Hermitian metrics with symmetry, J. Geom. Phys. 25 291-304 (1998).

[LP] Lüscher, M. and Pohlmeyer, K., Scattering of massless lumps and non-local charges in the two-dimensional non-linear $\sigma$-model, Nucl. Phys. B $13746-54$ (1978).

[MN] Mason, L.J. and Newman E.T., A connection between the Einstein and Yang-Mills equations, Communications in Mathematical Physics 121, 659-668 (1989).

[MW] Mason, L.J. and Woodhouse, N.M.J., Integrability Self-Duality, and Twistor Theory, London Mathematical Society Monographs New Series 15 (Oxford University Press, Oxford, 1996).

[NPT] Newman E.T., Porter J.R. and Tod K.P., Twistor surfaces and right-flat spaces, General Relativity and Gravitation 9, 1129-1142 (1978).

[O] Olver P.J., Applications of Lie Groups to Differential Equations, Graduate Texts in Mathematics 107 (Springer-Verlag, New York, 1986).

[Pa] Q.-H. Park, Integrable deformations of self-dual gravity, Physics Letters 269B, 271274 (1991),

[Pl] Plebański J.F., Some solutions of complex Einstein equations, Journal of Mathematical Physics 16, 2395-2402 (1975)

[Sa] Salamon, S., Quaternionic Kähler manifolds, Invent. Math. 67 143-171 (1982).

[S1] Strachan I.A.B., The symmetry structure of the anti-self-dual Einstein hierarchy, Journal of Mathematical Physics 36, 3566-3573 (1995).

[S2] Strachan, I.A.B., Hierarchy of conservation laws for self-dual gravity, Class. Quantum Grav. 10 1417-1423 (1993).

[W] Ward R.S., Integrable systems in twistor theory, in Twistors in Mathematics and Physics edited by Bailey T.N. and Baston R.J. (Cambridge University Press, Cambridge, 1990) pp 246-259. 